\title{
Control and Monitoring System for Composite Materials Fabrication Based on PPS Method
}

\author{
B. Sakowicz, D. Makowski, P. Sekalski, P. Marciniak, R. Kotas, G. Skrabalak, R. Talar, M. Rosinski, \\ and A. Napieralski
}

\begin{abstract}
Cubic boron nitride (cBN) is commonly used as a cutting tools material for the sake of its high hardness and wear-resistance. But on the other hand cBN, it requires application of an expensive HPHT (High Pressure High Temperature) to avoid the transformation of cBN into low-hardness $h-B N$, they must be sintered under a pressure of 5 to $6 \mathrm{GPa}$ which substantially increases their production costs. An alternative method, called PPS (Pulse Plasma Sintering), was proposed to decrease the production costs. The PPS significantly decreases the costs of production and therefore it is very attractive for industry. However, automation and dedicated control system for PPS is required to carefully control parameters and hamper the transformation of $\mathrm{cBN}$ into hBN. Only the application of automated production line guarantee high-quality of cubic boron nitride samples.

The paper discusses the requirements and presents the architecture of the control system dedicated for pulse plasma sintering system.
\end{abstract}

Index Terms-Pulse plasma sintering, cubic boron nitride, composite materials, real-time control system, SCADA, programmable logic controller.

\section{INTRODUCTION}

Diamond as a ultra-hard material is commonly used for a treatment of metal, plastic or building materials [1], [2]. The diamond-based cutting tools are mainly fabricated using sintered carbides composite material or PCD (PolyCrystalline Diamond) [3]. A good alternative to sintered materials is a cBN (Cubic Boron Nitride) that has a high hardness, wear-resistance and chemical neutrality [4], [5].

Sintered carbide composites enriched witch cubic boron nitride particles are ideal materials for cutting tools dedicated for metal treatment.

However cBN requires application of an expensive HPHT (High Pressure High Temperature) method for production

Manuscript received May 2, 2013; revised July 2, 2013.

This work has been supported by the project no PBS1/A5/7/2012 Application of the latest generation of DDCC and BNDCC composites in the field of cutting tool from the National Centre for Research and Development.

B. Sakowicz, D. Makowski, P. Sekalski, P. Marciniak, R. Kotas and A. Napieralski are with the Lodz University of Technology, Department of Microelectronics and Computer Science, Wolczanska 221/223, 90-924 Lodz, Poland (e-mail: sakowicz@dmcs.pl).

M. Rosinski is with Warsaw University of Technology, Faculty of Materials Science and Engineering, Woloska 141, 02-507 Warsaw, Poland (e-mail: ninmar@inmat.pw.edu.pl).

R. Talar is with Poznan University of Technology, Institute of Mechanical Engineering, Piotrowo 3, 61-138 Poznan, Poland (e-mail: rafal.talar@put.poznan.pl)

G. Skrabalak is with Institute of Advanced Manufacturing Technology, Wroclawska 37a, 30-011 Krakow, Poland (e-mail grzegorz.skrabalak@ios.krakow.pl). and therefore the tools fabricated with this method are at least ten times more expensive than sintered carbides [6], [7]. An alternative method for production of composite materials, based on cubic boron nitride with tungsten carbide-cobalt (cBN/WCCo) structure, was developed on the Faculty of Materials Science and Engineering, Warsaw University of Technology [8]-[10]. Composite samples can be manufactured within a few minutes using the PPS (Pulse Plasma Sintering) process in high vacuum $\left(10^{-3} \mathrm{mbar}\right)$ under a load lower than $100 \mathrm{MPa}$ and at a temperature of $1200^{\circ} \mathrm{C}$ [9].

Only sintering process with carefully controlled parameters allow to restrict the transformation of $\mathrm{cBN}$ into $\mathrm{hBN}$ and guarantee production high-quality cubic boron nitride samples. Thus a dedicated control systems for PPS is required to control parameters and therefore allows manufacturing samples and further improve the production process.

\section{LOW LEVEL CONTROL SySTEM}

An automated control and monitoring production process required in order to ensure reproducibility of the sintering processes using the PPS method. The described process is equipped with a low power control system. The system is mainly responsible for:

1) Subsystems control.

2) Acquisition and archiving of data from the sintering process monitoring sensors.

3) Various electrical circuits.

4) Subsystem protection.

5) Various process parameters modifications.

6) Communication with the user.

It is planned to use PLC (Programmable Logic Controller) as the main element of the control system. Data generated by process parameters monitoring sensors are sent with the use of the current logic (4-20 mA). Analogue signals are applied to an industrial converter, and then supplied to the PLC. Data from the sensors are processed by the central processing units. Data visualization and process control is done by use of the touch-screen industrial control panels. Parameter settings and test results will be stored in an external database and therefore will be available for further analysis. This solution allows to optimize the parameters during and after the production process.

One of the most important elements of the presented system is a dedicated database. It describes all of the subprocesses and conducted experiments with data mining mechanisms. This tool helps in the effective management and 
allows to develop production profiles.

There are two possible solutions of database implementation [11]:

1) Industrial database provided by the SCADA (Supervisory Control and Data Acquisition) software developer. It provides the ability to read and store all types of process data, operations and feeds the higher-level operations management systems with accurate, real-time information.

2) External database and a dedicated management application (made in technologies such as Java Enterprise Edition or Microsoft.NET) including mechanisms for full and partial synchronization with SCADA system.

\section{ARChITECTURE OF CONTROL SYSTEM}

The low-power control system is installed in industrial cabinets with safety circuits and control panel. Due to the presence of strong magnetic fields and electromagnetic interferences all equipment will be screened and reliable data transmission interfaces will be developed [12]. The discussed solutions will be implemented in software and hardware. During the design and validation of the system protocols will be developed and tested. This solution allows the transmission of data in an electromagnetic environment polluted by high-power circuits.

Project realization includes:

1) Preparation of the requirements for the control system and data acquisition.

2) Analysis and selection of technologies for the graphic user interface design.

3) Prototype system assembling.

4) Subsystems and entire system tests.

\section{A. Preparation of the Requirements for the Control System and Data Acquisition}

The requirements analysis is done according to the typical formula used in such instances. All of the required measured values and control subsystems were defined at the beginning of design process. Then, in an iterative process, the system diagram and graphic user interface are being implemented. The human machine interface will be designed in accordance with ergonomic rules, including minimum number of user actions leading to a desired result.

\section{B. Analysis and Selection of Technologies for the Graphic User Interface Design}

Supervision of the sintering process requires configuration and monitoring of a large number of process parameters. In order to facilitate the user to control and configure the device it is planned to use the SCADA software cooperating with low power control system.

Currently there are many SCADA technologies. The most popular solutions among them are:

1) Vijeo CITECT - Scheneider Electric.

2) EDS/ESS2 - Transition Technologies.

3) LabVIEW - National Instruments.

4) WinCC - Siemens.

5) Proficy HMI/SCADA - GE Intelligent Platforms.
The proposed control system is a soft real-time system. It is a medium size system (estimated number of inputs and outputs is more than 40). The redundancy of key components of the control system will increase its reliability.

The use of GE RX3i series controllers cooperating with Proficy HMI / SCADA software [13] enables quick design and subsequent adaptation of applications to control and monitor the process of sintering materials using plasma. Moreover, the system can be easily expanded by the operator panel (i.e. QuickPanel CE View), which enables a simple, tactile way to control the entire device clearly presenting process to the user.

\section{Prototype System Assembling}

The prototype system will be constructed in the laboratory of the Department of Microelectronics and Computer Science (DMCS) at Lodz University of Technology. In order to perform system tests emulator of other subsystems (providing sensor data) will be designed. This prototype allows to check the validity of the applied technology. In case of a positive validation the prototype will be extended with new functionalities.

The prototype will be used to develop control software and allow developing human machine interface. Working and tested low-power control system will be finally integrated with other subsystems.

At this stage it is planned to test:

1) The entire control system in the presence of a strong magnetic field.

2) Developed protocols for error-free data transmission (immunity to electromagnetic interference).

3) Protection circuits.

\section{Subsystems and Entire System Tests}

The system will be tested on many levels, including:

1) Single sensors and controls tests.

2) Integration tests allow advanced control schemes.

3) Functional tests allow the validation of the entire system.

4) Automatic tests allow to check the entire system after adding new features.

\section{CONTROL SYSTEM FOR COMPOSITE MATERIALS PRODUCTION}

\section{A. Of Programmable Logic Controller for Real-time Control}

The structure of the control system is hierarchical (Figure 1). It can be divided into three levels. The first is a system with a central PLC. Its task is to perform centralized supervision of the entire production process. The second level PLCs are responsible for the different subsystems of the industrial process (power supply, vacuum pump, system feeder).

Due to the different subsystems architecture Master-Multi Slave was chosen. The third level includes all the elements of diagnostic systems. All modules are equipped with sensors and measuring instruments.

The central controller (master) supervises the operation of the slave controllers:

1) High voltage power supply controller (HVPSC) - 
provides the ability to monitor and determine parameters of the UPS in real time. It is responsible for monitoring the technical condition of the power supply structure by using a complex system of test and measurement for detection, localization, identification and anticipation of the damage that may cause a malfunction of the power supply.

2) Vacuum system automatics - subsystem managed by a separate PLC consists of two locks and three diffusion pumps. The controller will be directly responsible for the digital control of the above elements and monitoring of their parameters.

3) Feeder subsystem - at this stage of the project the feeder type is not selected (robot arm revolving feeder, conveyor belt). However, for control of the subsystem responsible will also be a separate PLC. Its task is to control the operation of the feeder, and monitoring local sensors and signalling emergency situations.

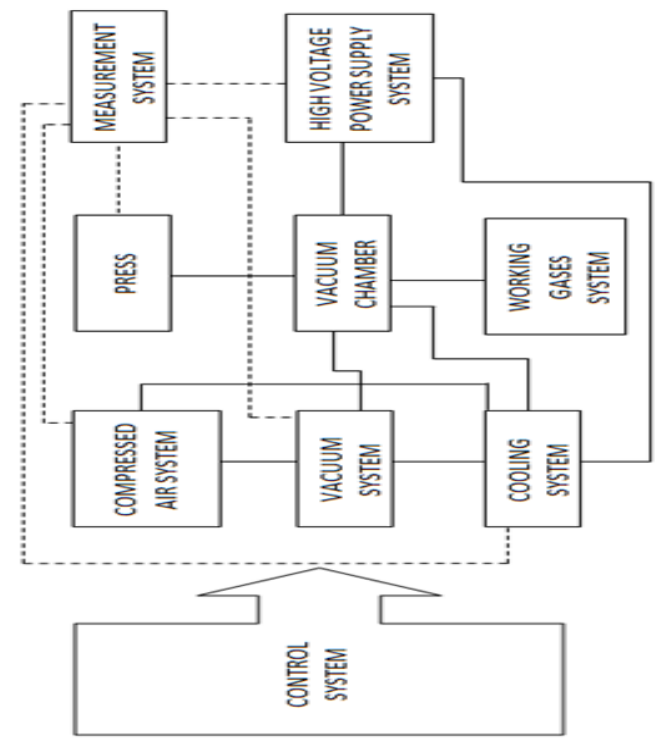

Fig. 1. Block diagram of composite materials production system.

The central PLC consists of [14]:

1) 40-Watt power supply that operates from an input voltage source in the range of 85 to 264 VAC or 100 VDC to 300 VDC. It provides This power supply provides three outputs:

$\bullet+5.1$ VDC output

$\bullet+24$ VDC relay output that can be used to power circuits on Output Relay modules

$\bullet+3.3$ VDC. This output is used internally by RX3i modules with IC695 catalogue numbers

2) Central processor unit (RX3i CPE305). It can be used to perform real time control of machines, processes, and material handling systems. It communicates with I/O and Intelligent Option modules over a dual PCI/Serial backplane. Main features:

- Contains 5 Mbytes of user memory and 5 Mbytes of non-volatile flash user memory

- Energy Pack, which on system power loss powers CPU long enough to write user memory to non-volatile storage (NVS)

- Configurable data and program memory

- RS-232 serial port
- Embedded Ethernet interface supports a maximum of two programmer connections.

3) Serial communications module, provides four independent, isolated serial ports. It can be located in the main PACSystems RX3i backplane. Each port can be configured for MODBUS Master, MODBUS Slave, Serial I/O protocol.

4) 24 VDC Positive/Negative Logic Input module provides 32 discrete input points. The inputs are positive or negative logic inputs and will operate at levels up to $30 \mathrm{~V}$. The inputs are arranged in four isolated groups of eight; each group has its own common. Isolation is provided between the four groups of inputs, however each group of eight inputs is referenced to the same user common connection.

5) 2 A relay output module provides 16 normally- open relay circuits for controlling output loads. The output switching capacity of each output is 2 Amps. The output points are in four groups of four points each. Each group has a common power output terminal. The relay outputs can control a wide range of load devices, such as: motor starters, solenoids, and indicators.

6) Universal analogue input module IC695ALG600 provides eight general purpose input channels and two Cold Junction Compensation (CJC) channels. Inputs are divided into two equal groups of four. Channels can be individually-configured using the Machine Edition software for:

- Any combination of up to 8 channels of voltage, current, thermocouple, RTD, and resistance inputs

- Resistance Inputs: 0 to 250 / 500 / 1000 / 2000 / 3000 / 4000 Ohms

- Current: 0-20 mA, 4-20 mA, +20 mA

- Voltage: $+50 \mathrm{mV},+150 \mathrm{mV}, 0-5 \mathrm{~V}, 1-5 \mathrm{~V}, 0-10 \mathrm{~V}$, $+10 \mathrm{~V}$

\section{B. User Interface}

A very important element of the control system is a dedicated operator panel. Visualization of the manufacturing process designed in SCADA system will be available to the operator through a touch panel. Ergonomic and intuitive interface will allow to modify any (or limited depending on access rights) process parameters. It is also considered to control the process from any computer equipped with the same SCADA visualization that is connected to the local network [15].

The central part of the SCADA system performs a dual role. On one hand, its purpose is to provide information about the system (indicator readings, equipment status) to the operator in a readable form. On the other hand, the SCADA system allows the operator to control the system and devices through modifications in accordance with specific procedures (settings and process parameters change).

The emphasis of this task is on the intuitive user interface. SCADA interface provides process visualization in the form of two-or three-dimensional technical drawing that are presented on a computer screen, which allows for easy and intuitive system control and management. In addition, the interface is designed in such a way as to minimize the number of steps performed in the operation. Convenient user 
interface is usually associated with greater sophistication of the system (the objective function is to minimize the number of necessary operations, not to minimize the application code), but incurred effort will yield higher production, which is crucial for each production process.

An important aspect of the control system is to ensure maximum safety for devices in the production line. SCADA system, by analyzing (in real time from) the current production process comparing them with pre-defined values, informs the operator about warning signals, alarms, existing risks and dangers.

The operator is responsible for fixing the problem. However some modern systems are able to automatically deal with multiple-existing atypical situations. The elimination of the human factor in such cases, increases the reliability and efficiency of the production process.

\section{CONCLUSION}

Pulse Plasma Sintering method requires a very complex control system. The system must be able to control and monitor several dozens of various parameters. In addition, the system must correctly respond to any emergency situations, expressed by the state of one or a combination of many parameters. The whole implementation is further complicated by the fact of different voltage levels and control devices from various manufacturers (which entails the need to deal with many different interfaces and protocols). According to the authors, the solution based on PLCs and SCADA allow for effective control of described industrial process.

\section{Plans FOR THE FUtURE}

The project will run until October 2015. The first stage is the construction of the prototype described in this paper. In the subsequent stages the prototype will be connected to the real device, where the series of tests will be applied. If successful, the system will be ready for a commercial deployment.

\section{REFERENCES}

[1] Anon, Ind. Dia. Rev, vol. 45, 1985, pp. 248.

[2] R. L. Sands and C. R. Shakespeare, "Powder metallurgy practice and applications," in Newnes, London, 1966, pp. 155.

[3] Q. S. Bai, Y. X. Yao, P. Bex, and G. Zhang, "Study on wear mechanisms and grain effects of PCD tool in machining laminated flooring," in Proc. Techn., vol. 22, pp. 111, 2004.

[4] L. Vel, G. Demazeau, and J. Etourneau, "Cubic boron nitride: Synthesis, physicochemical properties and applications," Mater. Sci. Eng. B, vol. 10, no. 2, pp. 149-164, 1991.

[5] G. P. Bogatyreva, V. M. Maevsky, E. R. Zusmanov, and A. V. Roitsin, "The effect of chemically active media on the structure and properties of cubic boron nitride," J. Solid State Chem, vol. 133, no. 1, pp. 292-295, 1997.

[6] A. Kurt and U. Seker, "The effect of chamfer angle of polycrystalline cubic boron nitride cutting tool on the cutting forces and the tool stresses in finishing hard turning of AISI 52100 steel," Mater. Des. 26 , pp. 351-356, 2005.

[7] J. A. Arsecularatne, L. C. Zhang, C. Montross, and P. Mathew, "On machining of hardened AISI D2 steel with PCBN tools," J. Mater. Process. Technol., vol. 171, pp. 244-252, 2006.
[8] A. Michalski and M. Rosiński, "Sintering diamond/cemented carbides by the pulse plasma sintering method," Journal of the American Ceramic Society, vol. 91, pp. 3560, 2008.

[9] A. Michalski, M. Rosiński, M. Płocińska, and J. Szawłowski, "Synthesis and characterization of cBN/WCCo composites obtained by the pulse plasma sintering (PPS) method," in Proc. IOP Conf. Series 202016 Symposium 14 Advanced Engineering Ceramicsand Composites: Materials Science and Engineering, 2011, pp. 18.

[10] M. Rosiński, A. Michalski, and J. Szawłowski "Sintered carbides composite material enriched with cubic boron nitride particles for cutting of hard-workable materials," Journal of Materials Science, 2011.

[11] J. Wu, Y. Cheng, and N. N. Schulz, "Overview of Real-Time database management system design for power system SCADA system," in Proc. of the IEEE, 2006, pp. $62-66$.

[12] A. H. Malik, M. A. Choudhry, T. Mehmood, and A. Hanif, "A generic procedure for troubleshooting of PLC based control systems," in Proc. Control Automation Robotics \& Vision, pp. $732-737$.

[13] Proficy HMI/SCADA, iFIX 5.5.pdf. [Online]. Available: http://www.ge-ip.com/download/proficy-hmi-scada-ifix-5-5-gfa-188 4/13112/3513/

[14] Control Systems Solutions Catalog. [Online]. Available: http://www.ge-ip.com (05.12 GFA-406H)

[15] F. Haji and L. Lindsay, "Practical security strategy for SCADA automation systems and networks," in Proc. Canadian Conference of Electrical and Computer Engineering., 2005, pp. 172 - 178.

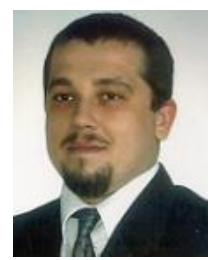

Bartosz Sakowicz was born in Lodz, Poland, on November 23, 1976. He received the M.Sc. and Ph.D. degrees from the Technical University of Lodz in 2001 and 2007, respectively. Currently he is with the Department of Microelectronics and Computer Science, Lodz University of Technology.

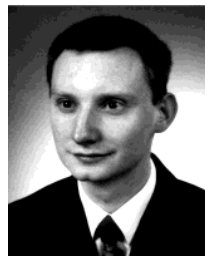

Dariusz Makowski received M.Sc. and Ph.D. degrees from the Lodz University of Technology in 2001 and 2006 , respectively. He is engaged in the studies and development of complex embedded and control systems. He is also involved in development of xTCA standards used in telecommunication and high-energy physics.

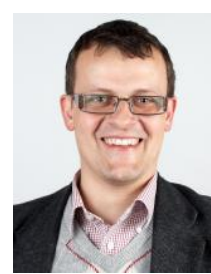

Przemyslaw Sekalski was born in Poland. He received a Master's Degree in Electronic and Telecommunication Engineering from the Lodz University of Technology in Poland in 2001, and Ph.D. Degree in Electronics in 2006 For 8 years he was working for High Energy Physics control systems at DESY in Hamburg, Germany. Currently, he is assistant professor at Lodz University of Technology and co-owner of Open-RnD, spin-off company which focus on embedded system design and mobile application development. Dr Sekalski main interest are in the field of dedicated sensor and actuators based networks with special focus on control and processing units.

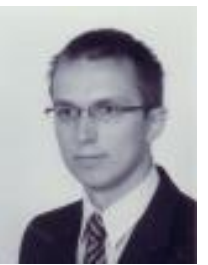

Pawel Marciniak received the M.Sc. degree from the Lodz University of Technology (LUT) in 2009 after defending his thesis entitled: "The use of SCADA System in Visualization of Medicine Production Process". He is a Ph.D. student of the Department of Microelectronics and Computer Science at LUT since September 2009.

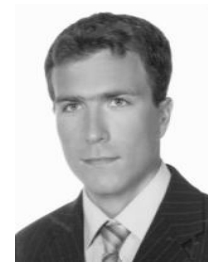

Rafal Kotas was born in Lodz, Poland, on August 31 1985. He received the M.Sc. degree from the Lodz University of Technology (LUT) in 2009 after defending his thesis entitled: "Data Glove Controlled by Microprocessor System". He is a Ph.D. student of the Department of Microelectronics and Computer Science at Lodz University of Technology since September 2009. 
Rafal Talar is a Ph.D. Eng. worker of the Poznan University of Technology, Institute of Mechanical Engineering since 2004. His work is focused in the field of engineering tribology. In the field of computer science he started in 1996 with his M.Sc thesis: "Computer aided calculations of technological conditions electrochemical - electrolytic cutting process".

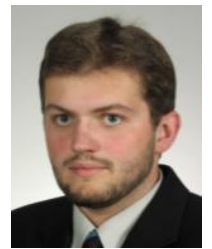

Grzegorz Skrabalak received his Ph.D. in 2013 from Warsaw University of Technology in the field of machine technology. He specializes in the non-conventional manufacturing methods (electrochemical, electrodischarge, hybrid), micromachining technologies and industrial automation (CNC machines and large scale automation systems).

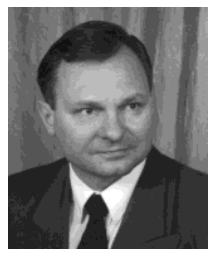

Andrzej Napieralski received his D.Sc. degree in electronics from the Warsaw University of Technology in 1989 as well as in microelectronics from the Universit'e de Paul Sabati'e in 1989. He is involved in an electrothermal simulation of VLSI circuits, modelling of transient thermal states in electronic circuits, thermal modelling of hybrid circuits and multidomain simulations. He is also engaged in microelectronics, microsystems and ASICs designing and simulation. 